\title{
Genetic resources and features of clonal micropropagation of Far Eastern species of Actinidia
}

\author{
Olga Molkanova ${ }^{1, *}$, Irina Krakhmaleva ${ }^{1}$, and Natalya Kozak $^{2}$ \\ ${ }^{1}$ Tsitsin Main Botanical Garden of Russian Academy of Sciences, 127276, 4, Botanicheskaya st., \\ Moscow, Russian Federation \\ ${ }^{2}$ Federal Horticultural Center for Breeding, Agrotechnology and Nursery, 115598, 4, Zagoryevskaya \\ st., Moscow, Russian Federation
}

\begin{abstract}
The unique collection samples of the Far Eastern species of the genus Actinidia Lindley preserved in Federal Horticultural Center for Breeding, Agrotechnology and Nursery were analysed. The in vitro collection of Actinidia has been formed since 2005 in Tsitsin Main Botanical Garden of Russian Academy of Sciences and currently includes more than 40 cultivars belonging to 3 species of Actinidia: A. kolomikta (Rupr. et Maxim) Maxim., A. arguta (Siebold et Zucc.) Planch. et Miq., and A.polygama (Siebold et Zucc.) Maxim. Easily reproducible, dependable method for clonal micropropagation of promising selected genotypes of Actinidia was optimized. It has been established regeneration features for the studied species of the genus Actinidia at the stage of micropropagation. A. kolomikta was characterized by a lower morphogenetic potential (multiplication coefficient 5.0) compared with other studied species. A. arguta and A. polygama were characterized by high multiplication coefficients, but no significant difference between them was present ( 8.0 and 7.7, respectively). The features of morphogenesis of male and female forms of $A$. kolomikta were revealed. The cultivars of the female forms had the highest multiplication coefficient ('Universitetskaya' 5.2, 'Prelestnaya' 5.2) compared with the male and bisexual forms ('Adam' 4.3 and 'Dr. Szymanowski' 4.3). The Quorin-Lepoivre nutrient medium supplemented with 6-Benzylaminopurine at a concentration of $0.5 \ldots 1.0 \mathrm{mg} \mathrm{L}^{-1}$ was found to be effective at the micropropagation stage, and the use of $\beta$-indole acetic acid at a concentration $0.5 \ldots 1.0 \mathrm{mg} \mathrm{L}^{-1}$ was better at the rooting stage.
\end{abstract}

\section{Introduction}

The genus Actinidia Lindley belongs to the family Actinidiaceae Van Tieghem /Hutchinson/. According to various classifications, there are from 40 to 76 species of Actinidia $[1,2]$. Most of this species are perennial woody lianas, which are distributed in tropical, subtropical and temperate regions of East Asia (China, Japan, and the Korean Peninsula). On the territory of Russia, Actinidia is located in the primary Amur-Ussuri

\footnotetext{
*Corresponding author: molkanova@mail.ru
} 
genetic center of origin of berry and fruit plants. The most winter-hardy species of Actinidia grow in the Russian Far East under natural conditions such as: Actinidia kolomikta (Rupr. Et Maxim) Maxim., A. arguta (Siebold et Zucc.) Planch. et Miq., A. arguta var. giraldii Diels., and A. polygama (Sieb. et Zucc.) Maxim [1].

The collection of Actinidia from the Federal Horticultural Research Center for Breeding, Agrotechnology and Nursery (FHRCBAN) preserved in the village of Mikhnevo, Moscow Region is the largest and most representative in Russia. The Institution contains about 200 samples, includes various species, cultivars and selected forms of national economic importance. At the same time, some valuable and unique genotypes in its composition are represented by single sample. Therefore, the use of modern biotechnological methods for the sustainable propagation of Actinidia gene pool is becoming more important. The development of effective methods of clonal micropropagation is the basis for the conservation of plant collections in vitro.

Both Russian and foreign scientists are actively carrying out research to study the influence of genetic features and components of the nutrient medium on the regenerative ability of explants, to increase the multiplication rate and obtain healthy planting material of representatives of the genus Actinidia [3-7].

This research aimed to describe collection samples of Actinidia and to study the features of clonal micropropagation of this culture.

\section{Methods}

For the study we used Actinidia plants from the FHRCBAN collection as a starting material. During the research process, samples were taken for subsequent in vitro cultivation.

The studies were conducted in the Laboratory of Plant Biotechnology of Tsitsin Main Botanical Garden of Russian Academy of Sciences (MBG RAS). The objects of the study were 10 cultivars of 3 Actinidia species:

1 - A. kolomikta: 'Adam' (male form), 'Dr. Szymanowski' (bisexual form), 'Universitetskaya', and 'Prelestnaya' (female forms);

2 - A. arguta: 'Bayern Kiwi' (male form), 'Velikansha', and 'Purpurnaya Sadovaya' (female forms);

3 - A. polygama: male form, 'Solntselikaya' and 'Zhar-Ptitsa' (female forms).

The research method was based on the generally accepted classical methods of work with cultures of isolated plant tissues and organs [8], and methods developed in the Laboratory of Plant Biotechnology of MBG [9].

At the micropropagation stage the nutrient medium QL (Quorin-Lepoivre, 1977) [10] was used with the addition of $30 \mathrm{~g} \mathrm{~L}-1$ sucrose, $6.8 \mathrm{~g} \mathrm{~L}^{-1}$ agar and $0.3 \ldots 1.0 \mathrm{mg} \mathrm{L}^{-1} 6-$ Benzylaminopurine (6-BAP) as the growth regulator. Hormone-free QL medium was used as a control.

The duration of subculture was 45 days, during the research. The following indicators were measured and calculated: multiplication coefficient, and spontaneous root formation $(\%)$.

The nutrient medium $1 / 2$ QL with the addition of $20 \mathrm{~g} \mathrm{~L}^{-1}$ sucrose, $6.8 \mathrm{~g} \mathrm{~L}-1$ agar, and different auxins: $\beta$-indole acetic acid (IAA) or $\beta$-indole butyric acid (IBA) at concentrations $0.5 \ldots 1.0 \mathrm{mg} \mathrm{L}^{-1}$ was used at the rooting stage.

The nutrient medium was sterilized using pressurized saturated steam $(\mathrm{P}=101 \mathrm{kPa})$ at $120^{\circ} \mathrm{C}$ for 20 minutes. The process of planting explants on a nutrient medium was carried out in a laminar box in accordance with the rules for working with sterile material. The culture conditions for the explants were: light intensity of $1.5 \ldots 2.0 \mathrm{Klux}, 16$-h photoperiod and temperature of $25 \pm 2{ }^{\circ} \mathrm{C}$. The experiments were carried out in 3 replicates, each with 10 
explants.

The results of the experimental data were processed statistically using the computer programs of the Microsoft Office Excel 2010 and PAST 2.17c. When determining the significant difference between the experimental variants, the least significant difference (LSD) at a level of $\mathrm{P}<0.05$ was used.

\section{Results}

The collection of Actinidia in FHRCBAN is the largest in Russia and has been formed since 1980 under the management of E.I. Kolbasina. The collection was formed as a result of the expeditions to the natural growth habitats of Actinidia in the Far East (in Primorye, on the Kuril Islands, Sakhalin Island) where the greatest genetic diversity is concentrated according to the academician N.I. Vavilov. In addition, samples were taken from cultivated plants in amateur and botanical gardens in the European part of the Russian Federation and Ukraine. One of these species, A. purpurea, was introduced from China. From 2008 and to the present time, further work on collecting samples and detailed study of biological and economically useful traits of selected forms has been continued by N.V. Kozak. Currently, 196 samples of 4 species of the genus Actinidia are preserved in the collection (Figure 1).

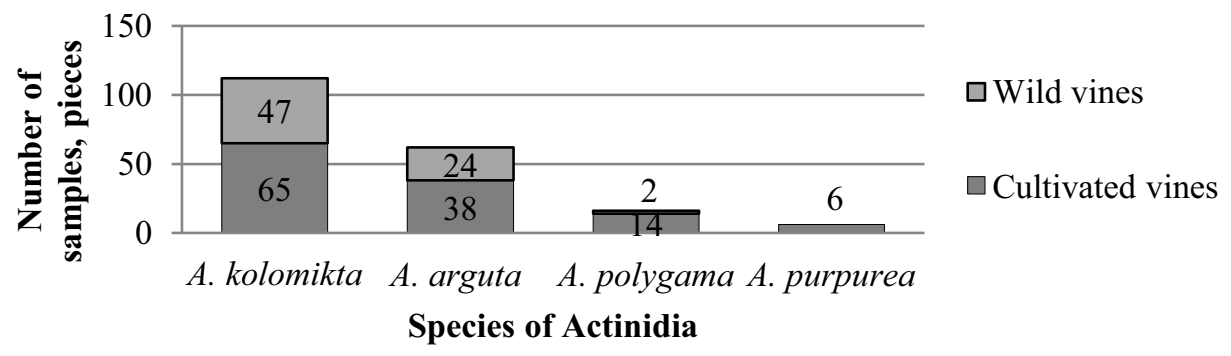

Fig. 1. Quantitative composition and origin of samples of the Actinidia collection in FHRCBAN.

The most representative in the collection is the A. kolomikta gene pool, which includes 112 samples. Other species are represented by smaller numbers: A. arguta 63, A. polygama 16, and A. purpurea 6 samples. Long-term observations have shown that plants of the above-mentioned Actinidia species have been successfully introduced in the conditions of the Moscow region.

Among the representatives of the genus Actinidia, the species A. kolomikta is the hardiest and the most widespread in Russian Federation. Breeding in Russia began with this species and of all the cultivars included in the State Register for Selection Achievements Admitted for Usage (State Register) of Russian Federation, 67\% (33 cultivars) belong to it [11]. The fruits of $A$. kolomikta are valued for a record high content of ascorbic acid (vitamin C from 1000 to $2200 \mathrm{mg} / 100 \mathrm{~g}$ of raw fruit weight or more) in combination with low acidity (usually does not exceed $3 \%$ ). It was found that $55 \%$ of the cultivars in the collection have a vitamin C content exceeding $1500 \mathrm{mg} / 100 \mathrm{~g}$. The highest content of vitamin C was found in the cultivars of 'Uslada' and 'Champion' (1850 and $2200 \mathrm{mg} / 100$ g). Dry matter accumulates in the fruits of 'Nadezhda' and 'Moma' more than others cultivars. These cultivars, unlike all others of this species, are not characterized by fruit falling off during ripening. In addition, A. kolomikta cultivars contain a large number of macro- and microelements in fruits. According to the amount of accumulation of macronutrients in fruits, among the cultivars of the collection, 'Dolgovechnaya', 'Uslada', 
and 'Vinogradnaya' are distinguished (42.068...45.638 mass \% in fruit ash), trace elements - in fruits of 'Pamyati Kolbasinoj' (4.904 mass \%) [12].

The species A. arguta and A. polygama are less common in Russia, due to their lower winter hardiness. New cultivars of $A$. arguta (such as 'Solnechnyj', 'Dachnaya', and 'Taezhnyj Dar') and A. polygama ('Dobryj Molodec', 'Abrikosovaya', and 'Krasna Devica') are characterized by greater winter hardiness, sufficient for growing in temperate climates without removed from supports and shelter for the winter. A. arguta is characterized by a higher yield compared to A. kolomikta and produce larger fruits (weighing up to $20 \mathrm{~g}$ or more) containing a lot of P activity substances (up to $55 \mathrm{mg} / 100 \mathrm{~g}$ of raw fruit weight). A. polygama, along with a high accumulation of ascorbic acid (up to $215 \mathrm{mg} / 100 \mathrm{~g}$ ), contains carotenoids (up to $6.4 \mathrm{mg} / 100 \mathrm{~g}$ ), including beta-carotene (provitamin A) in amounts comparable to the content in apricot and sea buckthorn fruits (2...6 mg / $100 \mathrm{~g}$ or more) [1]. The leaves of A. polygama also contain a high content of ascorbic acid (up to $690 \mathrm{mg} / 100 \mathrm{~g}$ of raw weight) and antioxidants, which allows them to be used as tea and food additives, the consumption of which can provide complex therapy [13].

The development, maintenance and replenishment of wild and cultivated plants is the most effective way of preserving and rational use of plant biological diversity. One of the effective ways to preserve it is to create genetic banks of plants in vitro. Based on a comprehensive study of the offspring of wild and cultivated forms of the genus Actinidia, the most promising ones were selected from the collection to study the features of clonal micropropagation and bank of meristems creation.

The in vitro collection of MBG RAS is a unique assemblage of the genus Actinidia representatives, reflecting the existing diversity of this culture in Russian Federation. The collection includes 42 cultivars of 3 species: A. kolomikta, A. arguta, and A. polygama. The most representative is the gene pool of $A$. kolomikta ( $50 \%$ of cultivars), compared with A. arguta (26\%), A. polygama (14\%) and A. arguta $\times$ A. purpurea (10\%). Most of the cultivars preserved and propagated in the in vitro collection were selected from the actinidia collection of FHRCBAN, which were bred by E.I. Kolbasina (64\%).

Before the in vitro system can be used to preserve the gene pool, effective methods of clonal micropropagation must be optimized. For in vitro cultivation of representatives of the genus Actinidia, breeding methods were chosen that minimize the risk of somaclonal variability. The main method which we use in reproduction is the activation of axillary meristems existing in plants, which allows the identity preservation of cultivated samples [14].

In the course of many years of the research, the sterilization scheme for the genus Actinidia representatives was optimized. Sterilizing agents were used to the following scheme depending on tissue types of initial explants: 2\% Fundazole solution with an exposure of 10 minutes, $70 \%$ ethanol for 30 seconds and $7 \%$ calcium hypochlorite with the addition of $1 \ldots 2$ drops of Tween-20 for $5 \ldots 7$ minutes. The nutrient medium MS (Murashige-Skoog, 1962) [15] containing $0.3 \mathrm{mg} \mathrm{L}^{-1}$ 6-BAP was used at the initiation stage. Some explants were cultured for 10 days on nutrient medium with the addition of the $0.25 \mathrm{mg} \mathrm{L}^{-1}$ antibiotic Gentamicin.

It was found that the development of microshoots of actinidia in vitro depends on the species (Figure 2). 


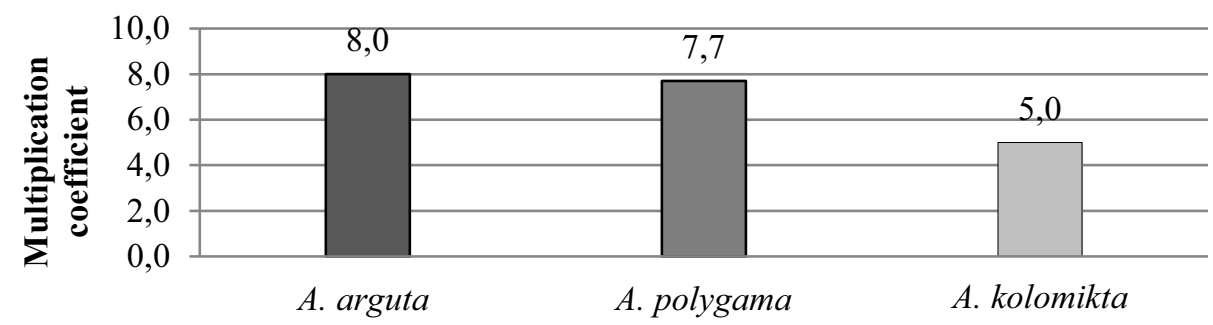

Species of Actinidia

Fig. 2. The influence of species characteristics on the multiplication coefficient of the genus Actinidia representatives (LSD05 1.1).

According to the morphogenetic potential, the studied species can be arranged in the following order: A. arguta (multiplication coefficient 8,0$)>A$. polygama $(7,7)>$ A. kolomikta $(5,0)$.

It was revealed the forms of $A$. kolomikta were different in their regenerative ability (Figure 3).

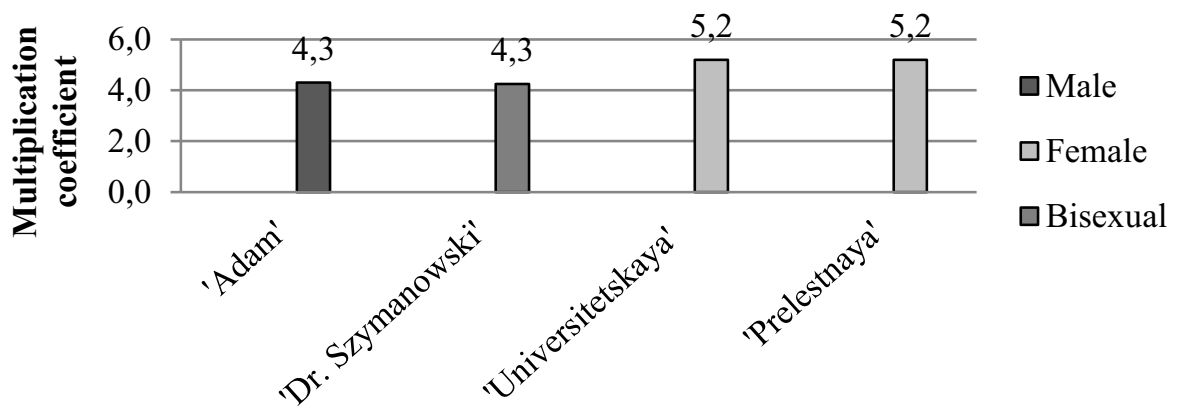

Cultivar

Fig. 3. The influence of forms characteristics on the multiplication coefficient of $A$. kolomikta (LSD05 0.4).

Analysis of the growth and development of male, bisexual and female forms of A. kolomikta showed that the highest rate of multiplication coefficient was found for female forms $(5,2)$. The male and bisexual forms had lower morphogenetic potential and there were no differences between them (multiplication coefficients 4.3).

The results showed the effect of 6-BAP concentration in nutrient medium on the regeneration potential of the studied species (Figure 4).

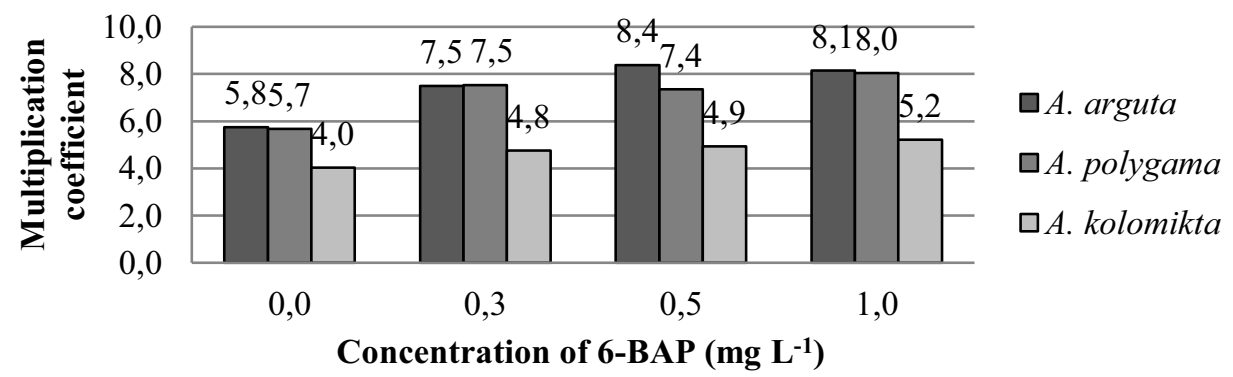

Fig. 4. The effect of the concentration of 6-BAP on the multiplication coefficient of the A. arguta (LSD 050.5 ), A. polygama (LSD05 0.6 ), and A. kolomikta (LSD $\operatorname{LS}_{05} 0.4$ ). 
When culturing $A$. arguta, the highest multiplication coefficient was obtained on the medium supplemented with 6-BAP at a concentration of $0.5 \ldots 1.0 \mathrm{mg} \mathrm{L}^{-1}$ (8.4 and 8.1, respectively). A. polygama had higher multiplication coefficient when cultured on medium with $1.0 \mathrm{mg} \mathrm{L}^{-1}$ 6-BAP (8.0). It was found that multiplication rate of $A$. kolomikta increased as the concentration of 6-BAP rose from $0.3 \mathrm{mg} \mathrm{L}^{-1}$ to $1.0 \mathrm{mg} \mathrm{L}^{-1}$ (4.8...5.2).

The process of root formation depended on auxin type and its concentration at the rooting stage. The highest percentage of rooted explants was observed on $1 / 2$ QL nutrient media with the addition of IAA (from $89 \%$ to $100 \%$ ) compared to IBA $(71 \ldots 92 \%$ ). Herewith, the optimal auxin concentration was $0.5 \mathrm{mg} \mathrm{L}^{-1}$ of IAA (Table 1).

Table 1. The effect of the concentration of auxins on the root formation of the genus Actinidia representatives.

\begin{tabular}{|l|c|c|c|c|}
\hline \multirow{2}{*}{ Species } & \multicolumn{4}{|c|}{ Rootability, \% } \\
\cline { 2 - 5 } & 0.5 IAA & 1.0 IAA & 0.5 IBA & 1.0 IBA \\
\hline A. kolomikta & 96 & 96 & 92 & 84 \\
\hline A. arguta & 96 & 89 & 71 & 71 \\
\hline A. polygama & 100 & 100 & 78 & 78 \\
\hline
\end{tabular}

Adaptation of microplants is the final stage of clonal micropropagation and one of the most costly and laborious. In our studies, actinidia regenerants were adapted on a substrate consisting of turf soil, peat, and perlite in a ratio of $2: 2: 1$ with the addition of sphagnum moss. It was found that the addition of sphagnum moss contributed to increasing the survival rate and minimizing the loss of plants during adaptation $(90 \% \ldots 100 \%$ of adapted plants).

As the results of the study, the methods of culture of the genus Actinidia representatives at the main stages of clonal micropropagation were optimized (Figure 5).
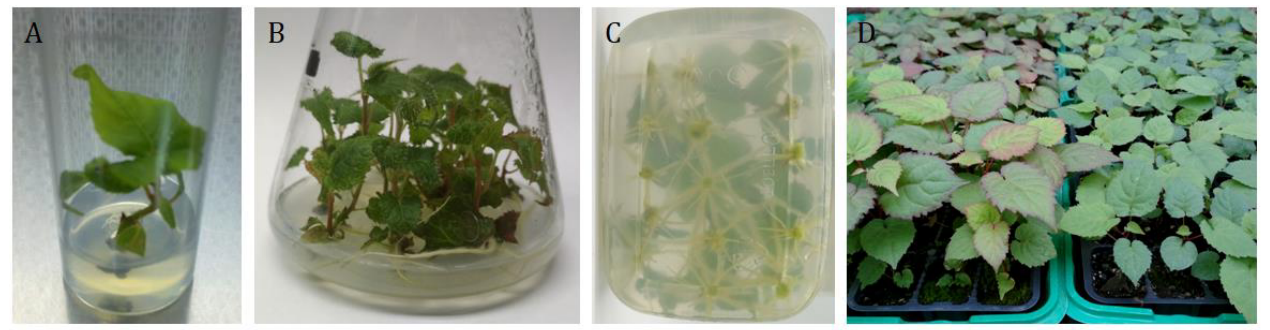

Fig. 5. A. kolomikta at the initiation stage (A) on the MS medium with the addition $0.3 \mathrm{mg} \mathrm{L}^{-1} 6-\mathrm{BAP}$, micropropagation stage $(\mathrm{B})$ on the QL medium $+1.0 \mathrm{mg} \mathrm{L}^{-1} 6-\mathrm{BAP}$, rooting stage $(\mathrm{C})$ on the $1 / 2$ QL medium $+0.5 \mathrm{mg} \mathrm{L}^{-1}$ IAA, and adaptation stage (D) on the soil substrate supplemented with sphagnum.

The development of a method for long-term preservation of regenerants has great importance in the technology of tissue organ cultures in vitro, which makes it possible to create a bank for valuable forms and rare plant cultivars. After studying the morphogenetic features and developing regeneration methods of actinidia to preserve the collection in vitro, microshoots $1-2$ metamers were cultured on $1 / 2 \mathrm{MS}$ culture medium containing $0.1 \mathrm{mg}$ $\mathrm{L}^{-1} 6$-BAP. The explants were subcultivated at a temperature of $25 \pm 2^{\circ} \mathrm{C}$, light intensity of 1.5...2.0 Klux, and 16-hour photoperiod. It has been established that the duration of subcultivation under such conditions can be 6 months. Currently, deposition conditions are being optimized by reducing the temperature. 


\section{Discussion}

Preservation of the plant gene pool is a global problem. Under the conditions of constant disruption of natural plant communities, there is a decrease in species diversity and the gene pool of valuable biological forms is reduced. Valuable phytoresource plants species are under threat of extinction. This also applies to representatives of the genus Actinidia, growing on the territory of the Russian Federation: A. kolomikta, A. arguta, A. arguta var. giraldii, and $A$. polygama. The main goal in the biodiversity preservation is a comprehensive study and conservation of genetic resources by supplementing and maintaining living plant collections, as well as operation modes of long-term preservation of explants in vitro, which ensure their viability and stability.

Fruit and berry crops are of great agriculture and economic importance. Many studies claim that the inclusion of fruits in the diet plays the greatest contribution in supplying human body with antioxidants and useful substances (carotenoids, phenolic compounds, sugars, acids, and others) [16, 17]. The advantage of actinidia fruits is due to their high content of biologically active substances with antioxidant properties, vitamins, catechins, pectin, tannins and coloring substances, flavonoids, alkaloids and other compounds. The use of fruits of this culture provides the human body with a complex of biologically active compounds $[18,19]$.

FHRCBAN bank maintains a collection of samples of Far Eastern Actinidia species in alive form. This Institution carries out breeding work to boost the most important economically valuable signs of culture: fruits non-shattering, size, high content of biologically active substances, good taste and hard winter resistance. Based on the selection of elite seedlings, 39 Actinidia cultivars were obtained (12 are protected by patents), which are included in State Register, which is $80 \%$ of all officially approved Actinidia cultivars in Russia [11]. Until now promising samples of Actinidia are also identified, which considered as a material source for breeding and introduction into agriculture.

Currently, for the preservation and stable reproduction of plant collections, it is relevant to use biotechnological techniques along with traditional methods. Meanwhile, the development of effective methods of clonal micropropagation is the basis of such studies. It is known that there is a complex of factors each of which individually and in combination with others has a significant influence on the development of cellular and tissue systems in vitro. Among them, the most important are the type of explant, the physiological state of donor plants, the conditions of plant cultivation in vitro, the composition of nutrient media. At the same time, the degree of influence of each of these factors depends on the genotype $[7,9]$.

Genotypic features of species and cultivars affect not only the micropropagation ability of the explant, but also its potential capacity for this process. At the micropropagation stage the explants of $A$. arguta and A. polygama developed more actively compared to A. kolomikta, which coincides with the results obtained by E.V. Malaeva (2020) [20]. It is worth noting that the regenerative ability of actinidia in in vitro culture corresponds to the growth peculiarities of these species in natural conditions.

The results of some studies on dioecious plant species showed that sexual dimorphism was clearly expressed in some woody plants and it was determined by certain morphological, anatomical, phenological-physiological and biochemical features. Skripchenko N.V. at al. (1999) was established the ratio of endogenous auxins, cytokinins, and abscisic acid on the annual shoots of Actinidia. The female forms of A. arguta and A. kolomikta have a higher coefficient of hormonal balance than male forms [21]. This was confirmed in our studies when cultivating various forms of A. kolomikta.

One of the important factors affecting in vitro propagation is the correct choice of growth regulators and their concentration. 6-BAP is well-known growth regulator for 
promoting cell division, cell growth, and differentiation of new shoots and generally used during at the micropropagation stage [22]. For effective clonal micropropagation of representatives of the genus Actinidia, 6-BAP has been previously used by other authors $[23,24]$. In our study, optimal concentrations of 6-BAP were established for the realization of morphogenetic potential of Far Eastern Actinidia species. It was found an increase in the multiplication coefficient of studied species with increasing concentration of 6-BAP from 0.3 to $1.0 \mathrm{mg} \mathrm{L}^{-1}$. At the micropropagation stage, QL medium supplemented with 6-BAP at the concentration of $0.5 \mathrm{mg} \mathrm{L}^{-1}$ was more effective for microshoot regeneration of $A$. arguta and A. kolomikta, and at a higher concentration $\left(1.0 \mathrm{mg} \mathrm{L}^{-1}\right)$ for $A$. polygama.

The genus Actinidia representatives are crops easily rooted without the use of auxins. The spontaneous root formation was observed on the studied species during culture process. After 45 days the percentage of spontaneous root formation was on average $96 \%$. Apparently, the internal regulation mechanisms of the rooting process, developed by species during the evolution process, were manifested. However, the use of auxins was the most effective method of the induction of root formation. A lot of research has been done to enhance rooting efficiency of the genus Actinidia representatives. Various auxin types and concentrations (IAA, IBA, and $\alpha$-napthalene acetic acid) were used for root induction at the rooting stage $[3,6,7,23]$. In this study, explants were rooted on a $1 / 2$ QL nutrient medium supplemented with auxins IAA and IBA after 14 days, and when use IAA at a concentration of $0.5 \mathrm{mg} \mathrm{L}^{-1}$ the rootability was the highest $(96 \ldots 100 \%$, respectively).

The conducted research allowed us to form in vitro gene bank of Actinidia species and cultivars as this is one of the most effective ways of plant biodiversity preservation.

\section{Conclusion}

The FHRCBAN collection maintains a unique assemblage of representatives of the genus Actinidia. However, some genotypes in their composition are represented by single sample. Development of propagation and preservation methods of unique forms and plant cultivars of Actinidia allows to expand genetic diversity and improve the range of cultivated genotypes. Morphogenesis and regeneration in vitro depend on several factors, and there are no general biotechnological methods for propagation and preservation plant taxa. In this study, it was found that the development of Actinidia explants depended both on species and sex of plants, and on the composition nutrient medium. The cultivars of A. kolomikta were characterized by a lower regeneration ability compared to A. arguta and A. polygama. A comparative analysis of various forms of $A$. kolomikta showed that the female forms had higher multiplication coefficient than male and bisexual forms. At the stage of micropropagation of the studied species, QL nutrient medium supplemented with 6-BAP at a concentration of $0.5 \ldots 1.0 \mathrm{mg} \mathrm{L}^{-1}$ effectively influenced, and the use of IAA at a concentration $0.5 \ldots 1.0 \mathrm{mg} \mathrm{L}^{-1}$ was better at the rooting stage.

It was found that the collection of Actinidia in vitro can be successfully maintained for 6 months at a temperature of $25 \pm 2{ }^{\circ} \mathrm{C}$, light intensity of $1.5 \ldots 2.0 \mathrm{Klux}$ and 16 -hour photoperiod.

The work was carried out in accordance to Institutional research project №18-118021490111-5.

\section{References}

1. E.I. Kolbasina, L.V. Solovyova, N.N. Tulnova, N.V. Kozak, N.V. Skripchenko, P.A. Moroz, N.A. Korchemnaya, A.I. Gvozdeczkaya, Cultured Flora of Russia: Volume Actinidia, Schisandra (Rossel'khozakademiya, Moscow, 2007) 
2. A.R. Ferguson, H. Huang, Horticultural reviews, 33 (2007)

3. A. Nasib, K. Ali, S. Khan, Pak. J. Bot., 40, 6 (2008)

4. R. Hameg, T.A. Arteta, P.P. Gallego, M.E. Barreal, Acta Hortic., 1218 (2018)

5. R. Hameg, T.A. Arteta, M. Landin, P.P. Gallego, M.E. Barreal, Front. Plant Sci., 11, 2088 (2020)

6. S. Purohit, J.M.Rawat, V.K. Pathak, D.K. Singh, B. Rawat, In Vitro Cell. Dev. Biol. Plant, 57, 3 (2021)

7. E.V. Malaeva, O.I. Molkanova, Acta Hortic., 1324 (2021)

8. R.G. Butenko, Biology of higher plant cells in vitro and biotechnology based on them (FBK-PRESS, Moscow, 1999)

9. O.I. Molkanova, O.V. Koroleva, T.S. Stacheeva, I.L. Krakhmaleva, E.A Meleshchuk, Achievements of science and technology of AIC, 32, 9 (2018)

10. M. Quoirin, P. Lepoivre, Acta Hortic., 78 (1977)

11. State Register for Selection Achievements Admitted for Usage (National List) (FGBNU "Rosinformagrotekh", Moscow, 2021)

12. N.V. Kozak, S.M. Motyleva, D.V. Panishcheva, Z.A. Imamkulova, I.M. Kulikov, S.M. Medvedev, Horticulture and viticulture, 3 (2021)

13. M.S. Gins, V.K. Gins, A.A. Baykov, V.F. Pivovarov, N.V. Kozak, Z.A. Imamkulova, I.M. Kulikov, S.M. Medvedev, E.M. Gins, Vestnik of the Russian agricultural science, $1(2018)$

14. Yu.N. Gorbunov, O.I. Molkanova, D.A. Egorova, I.V. Shirnina, O.G. Vasilyeva, «AgroEcoInfo», 44, 2 (2021)

15. T. Murashige, F.Skoog, Physiol. Plant., 15 (1962)

16. H. Wang, G. Cao, R.L. Prior, J. Agric. Food Chem., 44 (1996)

17. A. Zulueta, M.J. Esteve; A. Frígola, Food Chem., 114, 1 (2009)

18. S.M. Motyleva, N.V. Kozak, I.M. Kulikov, Problems of Biological, Medical and Pharmaceutical Chemistry, 21, 10 (2018)

19. D. Panishcheva, S. Motyleva, N. Kozak, Potr. SJF Sci., 15 (2021)

20. E.V. Malaeva, Agricultural Science Euro-North-East, 19, 2 (2020)

21. N.V. Skripchenko, L.I. Musatenko, P.A. Moroz, V.A. Vasjuk, Plant Introduction, 2 (1999)

22. E.F. George, M.A. Hall, G.J. De Klerk, Plant propagation by tissue culture (Springer, Dordrecht, 2008)

23. F.A. Akbas, C. Isikalan, S. Namli, D. Basaran, Int. J. Agric. Biol., 9, 3 (2007)

24. T.A. Arteta, R. Hameg, M. Landin, P.P. Gallego, M.E. Barreal, Eur. J. Hortic. Sci., 83, $4(2018)$ 